Lynden A. Archer

Dennis Ternet

Ronald G. Larson

\section{"Fracture" phenomena in shearing flow of viscous liquids}

in polystyrene, bubbles open up within the sample, as occurs in cavitation. Some similarities are pointed out between these phenomena and that of "lubrication failure" reported in the tribology literature.

Key words Fracture - cavitation

\author{
L.Y. Archer \\ Department of Chemical Engineering \\ Texas A\&M University \\ College Station, Texas 77843-3122, USA \\ D. Ternet \\ Department of Chemical Engineering \\ University of California \\ Santa Barbara, California 93106, USA \\ Prof. Dr. R. G. Larson (『) \\ Department of Chemical Engineering \\ University of Michigan \\ Ann Arbor, Michigan 48109-2136, USA \\ E-mail: rlarson@engin.umich.edu
}

\begin{abstract}
In start-up of steady shearing flow of two viscous unentangled liquids, namely low-molecucose, the shear stress catastrophically collapses if the shear rate is raised above a value corresponding to a critical initial shear stress of around $0.1-0.3 \mathrm{MPa}$. The time dependence of the shear stress during this process is similar for the two liquids, but visualization of samples in situ and after quenching reveals significant differences. For $a$-D-glucose, the stress collapse evidently results from debonding of the sample from the rheometer tool, while
\end{abstract}

example, by meniscus "edge fracture", which can be driven by the influence of the second normal stress difference (Lee et al., 1992). However, in the experiments of Chen et al., one of the melts was a polystyrene of low enough molecular weight to be unentangled, and so unlikely to slip against the rheometer tools, at least by the slip mechanisms currently thought to be important. Unmagnified in situ observations of the polystyrene sample during the waveform decay showed what appeared to be bubbles or holes opening up in the sample, especially near its edge. After cessation of shearing, the bubbles slowly redissolved over the course of a few hours.

In this brief report, we examine analogous stress-decay, or "fracture," phenomena in steady shearing flows of an unentangled polystyrene melt of molecular weight 13000 , as well as a melt of $a$-D-glucose, which has a molecular weight of only 180 Daltons, and so is not even polymeric. High stresses can be obtained in both 
of these liquids at modest shear rates because they are very viscous (viscosities of $10^{4}-10^{6} \mathrm{~Pa} \mathrm{~s}$ ) at temperatures in the vicinity of their respective glass transitions.

\section{Experimental}

Polystyrene of molecular weight 13000 (PS13K) and polydispersity $\left(M_{w} / M_{n}\right)$ of 1.06 was obtained from the Pressure Chemical company. It was heated to $110^{\circ} \mathrm{C}$ under vacuum for a period of several days to produce degassed, transparent, disks for rheological investigation. $a$-D-glucose, 96\% anhydrous, obtained from Aldrich, is crystalline at equilibrium below its melting point of $156^{\circ} \mathrm{C}$. If, however, it is quenched rapidly, it can be supercooled down to a glass transition temperature $T_{g}$ of $38^{\circ} \mathrm{C}$ where it vitrifies. Using this method, one can produce transparent, non-crystalline, $a$-D-glucose disks for rheological investigation. When these disks are heated in the rheometer to temperatures of up to $25^{\circ} \mathrm{C}$ above $T_{g}$, they become transparent viscous liquids.

Rheological measurements were made uisng a Rheometrics System 4 rheometer, equipped with an $8-\mathrm{mm}$ diameter aluminium cone-and-plate fixture with a cone angle of 0.1 radians that allows stresses in excess of $1 \mathrm{MPa}$ to be measured conveniently. In some cases (described below), a coating of tetrafluoroethylene (Teflon) was applied to the aluminum surfaces by first cleaning them thoroughly with methylene chloride and water and then spraying on the coating (Dynamar) dissolved in a volatile solvent. The coated surface needed to be baked at $100^{\circ} \mathrm{C}$ for around an hour to obtain durable coatings.

Since the System 4 uses a convection oven, temperatures are accurate only to within a degree or two. Because of the strong temperature-sensitivity of the viscosities of these liquids in the temperature ranges of interest, the viscosities reported here probably have an accuracy of no greater than a factor of two or so. Errors are not likely to be much larger than this, since the viscosity reported here for PS13K is consistent with that measured on a polystyrene of almost identical molecular weight (13300) by Allen and Fox (1964), who used a capillary viscometer with much better temperature control than we could obtain. In addition, we shall see that the critical stresses for "fracture" are not so temperature sensitive, and are therefore more accurate than the viscosity values.

In situ visualization experiments were performed using Cambridge Shearing System made by Linkam in collaboration with the Department of Chemical Engineering at Cambridge University. It consists of two parallel glass disks which are in thermal contact with a silver-block electric heater, mounted on the stage of a microscope. The bottom window is attached to a metal disk which is made to rotate by a stepper motor in either steady or oscillatory rotation. Openings in the block permit transmission of light through the sample for microscopic examination of it under shear. The window is $2.5 \mathrm{~mm}$ in diameter and is centered at a position $7.5 \mathrm{~mm}$ from the axis of rotation, close to the edge of the sample. Steady shearing rates were kept below $5 \mathrm{~s}^{-1}$, low enough to avoid inertially driven secondary flows and viscous heating.

\section{Results}

Figure 1 shows transient shear stresses for the two liquids, highlighting three different shear rates for each. At low shear rates (curve 1), in relatively short time periods the shear stresses attain steady-state values that
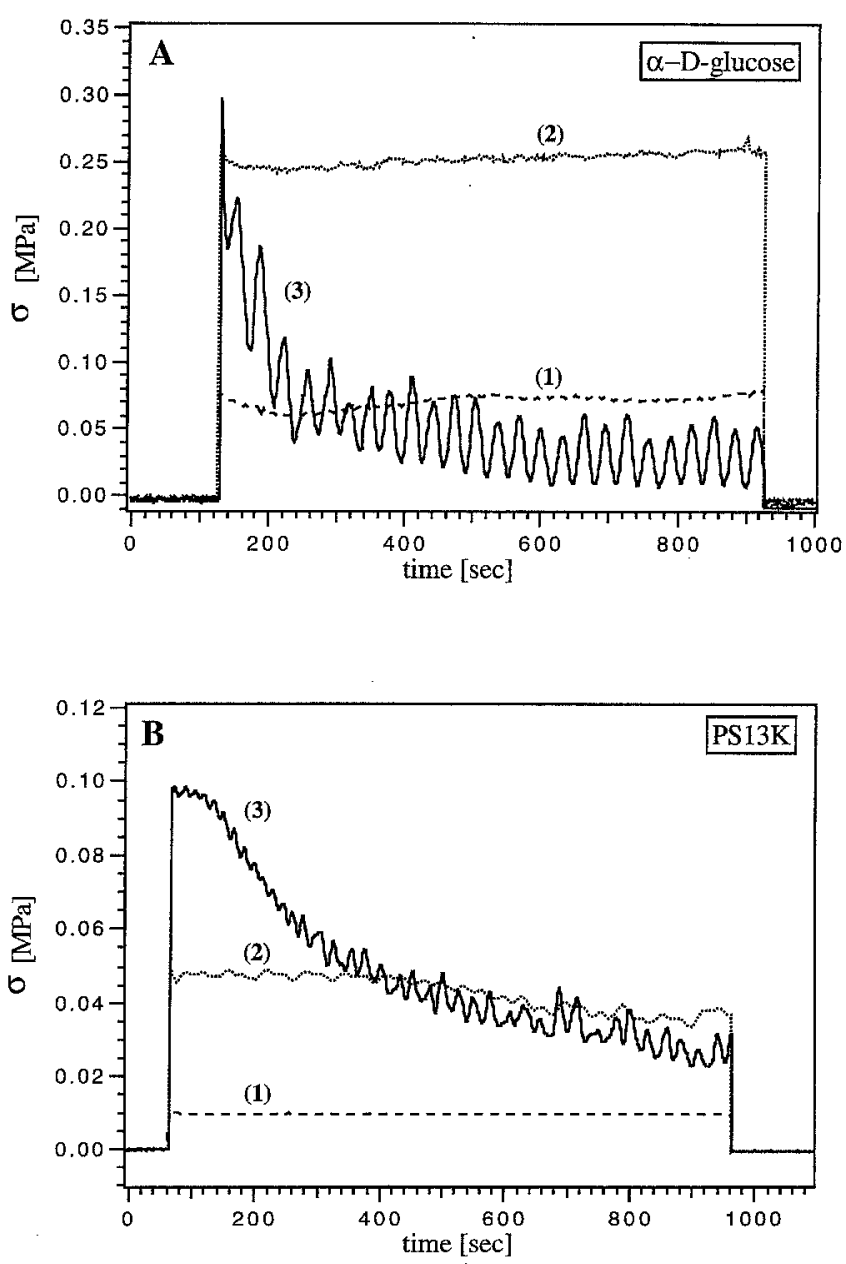

Fig. 1 A) Representative transient shear stresses for $a$-D-glucose at $60^{\circ} \mathrm{C}$, showing three flow regimes: 1) steady state, 2) weakly oscillatory and 3) gross stress decay superimposed on oscillations. B) The same as A), but for PS13K at $127.5^{\circ} \mathrm{C}$ 

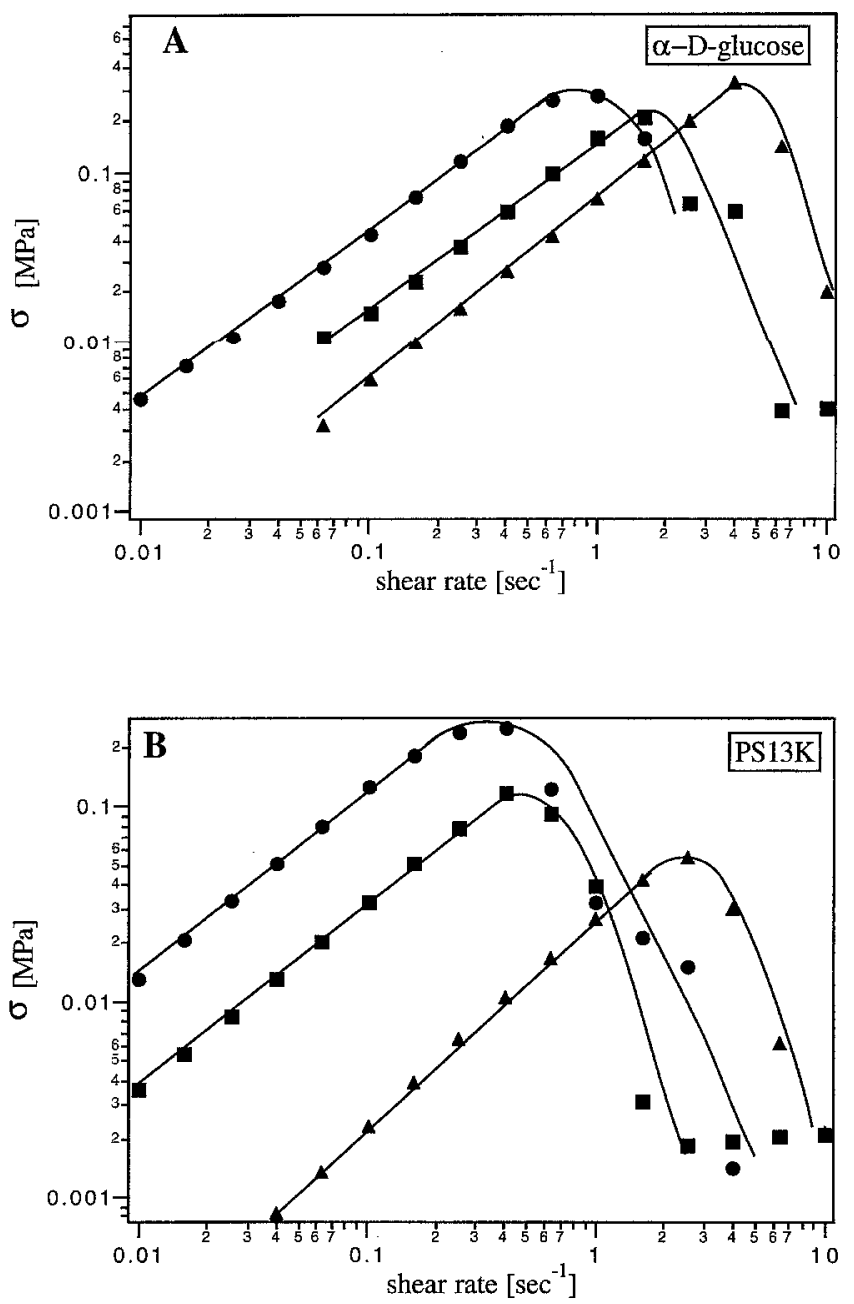

Fig. 2 A) Steady-state shear stress of $a$-D-glucose as a function of shear rate at $T=54.6^{\circ} \mathrm{C}$ (circles), $57.4^{\circ} \mathrm{C}$ (squares), and $60.0^{\circ} \mathrm{C}$ (triangles). B) The same as A), but for PS13K at $T=116.5^{\circ}, 127.5^{\circ}$, and $134.5^{\circ} \mathrm{C}$ (triangles)

are linear in the shear rate (Fig. 2); the fluid viscosities are therefore Newtonian (Fig. 3). At higher shear rates (curve 2 in Fig. 1), steady-state shear stresses are achieved only for short periods of time, after which they oscillate in time. Figure 1 shows that while these oscillations are pronounced for PS13K, they are weaker for $a$-D-glucose. The oscillation frequencies do not match any harmonic of the angular velocity of the rotating cone, and therefore do not appear to result from instrumental artifact. At slightly higher shear rates (curve 3 of Fig. 1), the shear stress for $\alpha$-D-glucose collapses dramatically with time, and after prolonged shearing finally achieves a non-decaying, but still oscillatory, state. The final time-averaged value of the stress is about a decade lower than its value a few seconds after inception of flow. The shear stresses for PS13K do not reach steady state within the duration of the experiments $(900 \mathrm{~s})$. During the stress decay, oscillations of
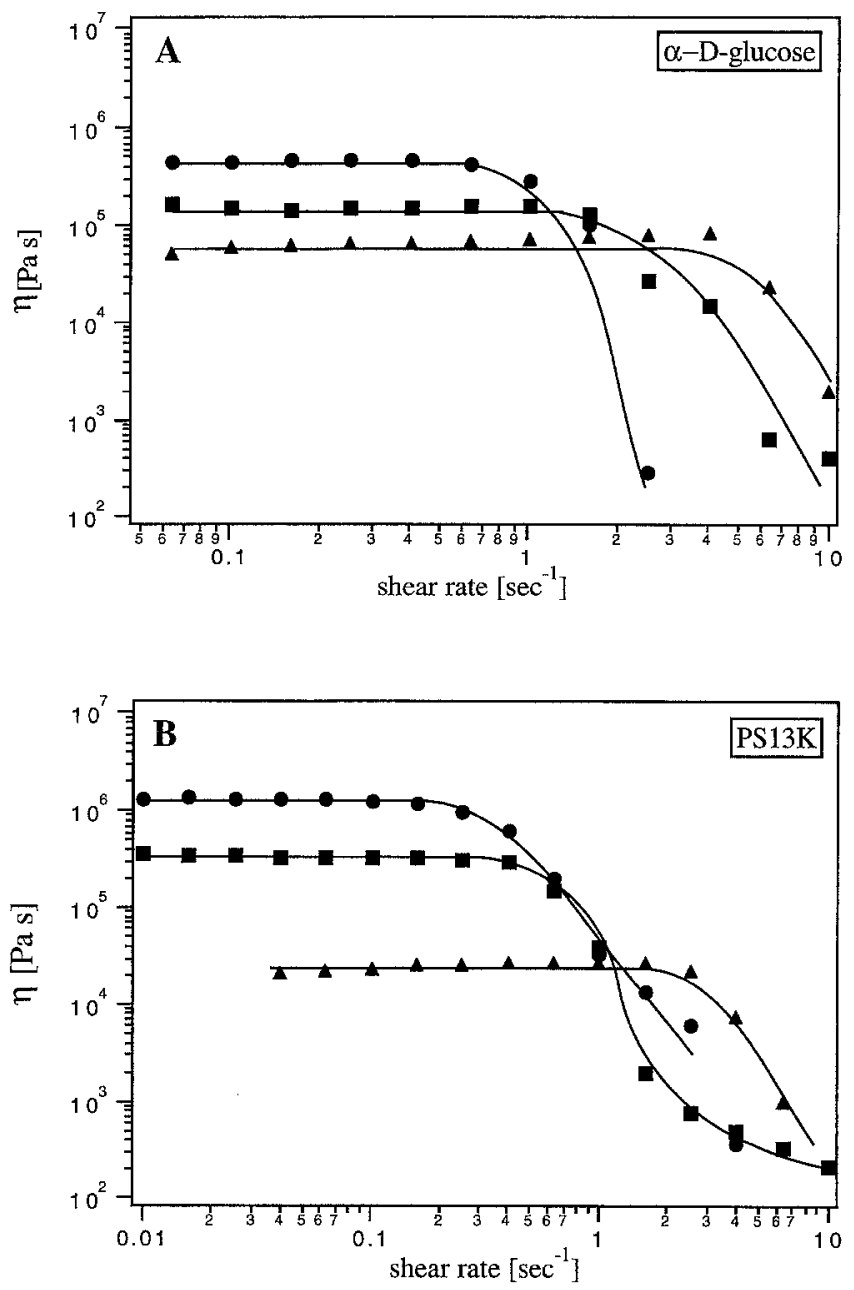

Fig. 3 A) Shear viscosity of $a-D$-glucose at various temperatures exceeding $T_{g}$. The symbols have the same meaning as in Fig. 2. This plot shows that at each temperature the viscosity is Newtonian up to the shear rate of failure. B) The same as A), but for PS13K

enhanced amplitude are observed. The frequencies of the oscillations are weak functions of shear rate and their amplitudes are as high as $50 \%$ of the mean stress for $a$-D-glucose samples, and about $10 \%$ of the mean for PS13K.

The dramatic collapse of the shear stress above a critical steady rate of shear is qualitatively similar to that observed in oscillatory flow of low molecularweight polystyrene (Chen et al., 1994). The oscillations observed during steady shear flow, if superimposed onto the stress waveform produced during oscillatory flow, might account for the stress-waveform distortion observed in the latter.

When final time-averaged values of the shear stress for $a$-D-glucose and long-time values for PS13K are plotted against shear rate in Fig. 2, one finds a maximum in the shear stress. We define this maximum to be the critical stress $\sigma_{c}$; it is around $0.3 \mathrm{MPa}$ for $\alpha$-D-glu- 


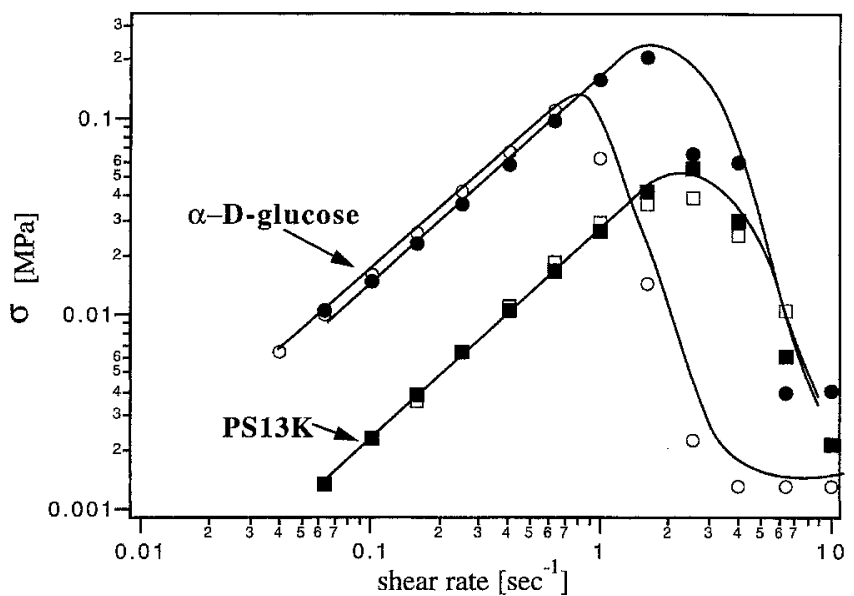

Fig. 4 Steady-state shear stresses for $a$-D-glucose at $57.4^{\circ} \mathrm{C}$ (circles) and PS13K at $134.5^{\circ} \mathrm{C}$ (squares) at various shear rates. Data represented by the open symbols are from experiments in which the aluminum fixtures were coated with tetrafluoroethylene films and the filled symbols represent data from similar experiments using bare aluminum fixtures

cose and 0.1 MPa for PS13K at the temperatures considered. Furthermore, the viscosities of both fluids plotted in Fig. 3 are almost shear-rate independent, up to $\sigma_{c}$.

We also measured the first normal stress difference $N_{1}$ for PS13K; it shows a similar collapse in stress above the critical shear rate. Below the critical condition $N_{1}$ is quadratic in shear rate, and can therefore be represented by

$$
N_{1}=K \sigma^{2}
$$

where $K$ is a constant, $K \approx 5(\mathrm{MPa})^{-1}$. From the Rouse theory for unentangled melts, the value of $K$ is given by (Larson 1988)

$$
K=\frac{S_{2}}{S_{1}^{2}} \frac{2 M}{N_{A} k T \rho}
$$

where $S_{2} \equiv \sum_{i}\left(\lambda_{i} / \lambda_{1}\right)^{2} ; S_{1} \equiv \sum_{i} \lambda_{i} / \lambda_{1}$, and the $\lambda_{i}$ 's are the Rouse relaxation times, $M$ is the polymer molecular weight, $N_{A}$ is Avogadro's number, and $\rho$ is the liquid density. For the Rouse model, $S_{2} / S_{1}^{2}=0.4$. Taking $\rho=1.0 \mathrm{gm} / \mathrm{cm}^{2}$, we obtain a theoretical value of $K=3.1(\mathrm{MPa})^{-1}$, in reasonable agreement with the measured value of $5(\mathrm{MPa})^{-1}$. For $a$-D-glucose, $N_{1}$ is zero to within experimental noise, as expected for a small-molecule liquid. The $N_{1}$ measurements show that PS13K is a viscoelastic fluid, as expected for a polymer melt, while $a$-D-glucose is Newtonian under the conditions studied here.

We also measured the linear viscoelastic properties of both $a$-D-glucose and PS13K in small-amplitude oscillatory shearing. For PS13K, the critical shear rate

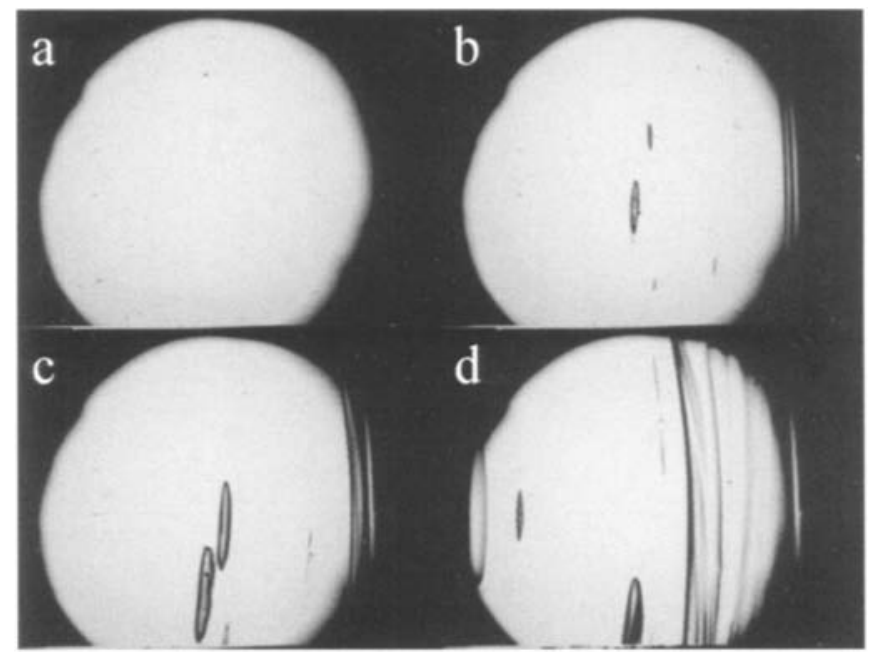

Fig. 5 Images from the Cambridge flow cell of a polystyrene sample undergoing steady shear at a shear rate $\dot{y}=1.0 \mathrm{~s}^{-1}$ and a gap of $800 \mu \mathrm{m}$ at $134^{\circ} \mathrm{C}$. The sample is viewed along the flow gradient direction from above and the line at the right side of the image is the edge meniscus. The lower disk rotates clockwise, which in this figure is top to bottom. The field of view is $2.5 \mathrm{~mm}$ wide. a) $0 \mathrm{~s}$ (start of shearing); the sample contains some dust particles but no bubbles; b) $9 \mathrm{~s}$; small elongated bubbles open up and the edge meniscus moves into view at the right edge of the field of view; c) $11 \mathrm{~s}$; the bubbles grow larger and the meniscus moves further inward; d) $24 \mathrm{~s}$; the bubbles reach an average steady state size and concentration, and the edge meniscus becomes very broad

is somewhat outside the terminal region, close to the frequency at which high-frequency "glassy modes" become active while for $a$-D-glucose, the critical shear rate is well within the terminal zone, a decade below the transition to higher frequency modes. Like the normal-stress data, these linear-viscoelastic data show that the "fracture" phenomenon for $a$-D-glucose is strictly within the non-elastic range for that liquid, while for the polystyrene samples it occurs within the viscoelastic regime, although the viscosity of the polystyrene is still within its Newtonian-plateau region.

After completion of a steady shearing experiment on $a$-D-glucose, the sample could be quenched to room temperature, removed intact from the rheometer, and inspected. The disks of $a$-D-glucose removed from the rheometer were transparent and showed no indication of any flow-induced crystallization, nor of any flow-induced irregularities in sample shape, such as "edge fracture". During removal, it was observed that samples that had been subjected to shearing stresses in excess of $\sigma_{c}$ had become debonded from one of the fixtures, usually the upper cone. This suggests that fracture or delamination had occurred at this surface. Further evidence of delamination or perhaps slip at the wall is inferred from the observation that the critical stress $\sigma_{c}$ could be decreased by a factor of about two by applying a film of Teflon onto the bare aluminum surfaces 


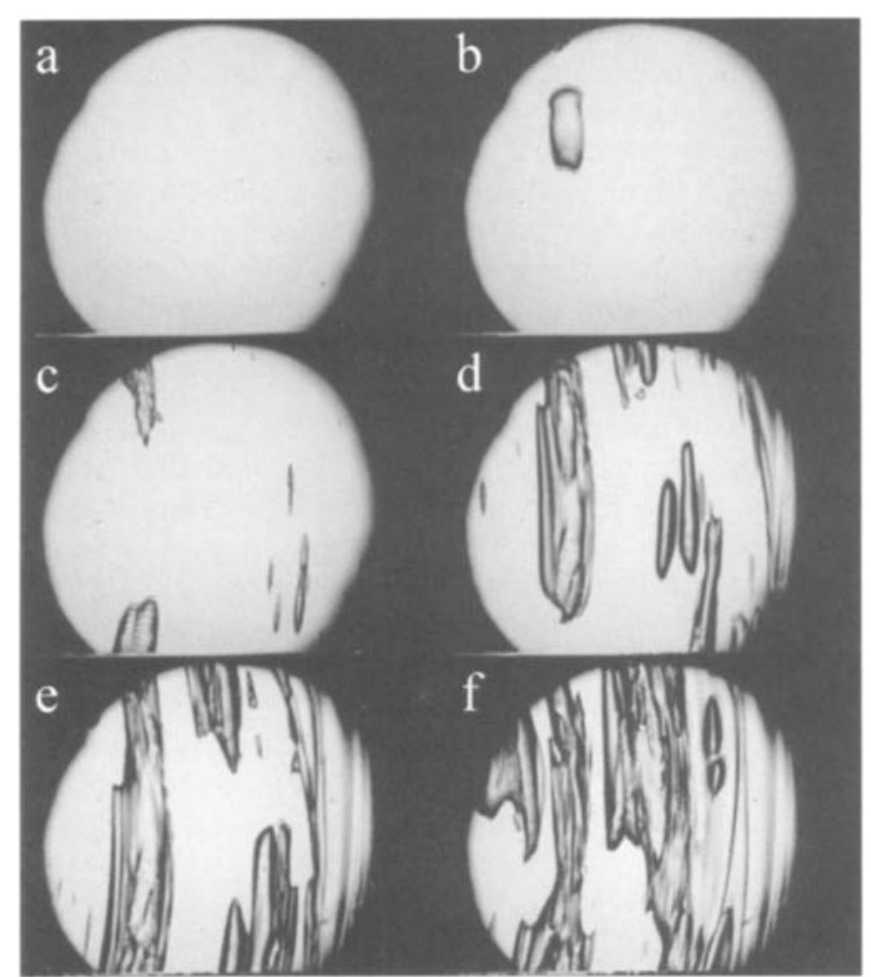

Fig. 6 As in Fig. 5, except that the shear rate is $2.5 \mathrm{~s}^{-1}$. The sample edge meniscus is out of the field of view to the right. a) $0 \mathrm{~s}$; start of shearing; b) $3 \mathrm{~s}$; a large bubble appears; c) $4 \mathrm{~s}$; bubbles multiply; d) $6 \mathrm{~s}$; e) $7 \mathrm{~s}$; f) $9 \mathrm{~s}$; most of the sample is disrupted by bubbles and air pockets

(see Fig. 4). The critical stress for polystyrene PS13K, on the other hand, showed little or no sensivity to this surface treatment (again see Fig. 4).

For PS13K, it was not possible to separate the cone and plate from the sample after shear without damaging it. Further qualitative studies of the polystyrene samples were therefore carried out in the Cambridge Shearing Cell at $T=134^{\circ} \mathrm{C}$ and a gap of around $800 \mu \mathrm{m}$. In this cell, we observed nothing unusual for shear rates less than $0.4 \mathrm{~s}^{-1}$. For $0.4 \leq \dot{\gamma} \lesssim 2.0 \mathrm{~s}^{-1}$, bubbles occasionally opened up in the sample during shearing (see Fig. $5 \mathrm{a}-\mathrm{d}$ ) and would sometimes close up again. These bubbles were located well away from the sample's edge meniscus. However, the meniscus at the sample's edge also broadened and sometimes developed a ragged appearance. At a critical shear rate of $2.5 \mathrm{~s}^{-1}$, immediately after onset of shearing, huge bubbles opened up, and rapidly multiplied within the bulk of the sample, well away from the sample edge (see Fig. $6 \mathrm{a}-\mathrm{f}$ ). This completely destroyed the integrity of the sample, no doubt leading to the massive stress decay seen in Fig. 1. Note in Fig. $2 \mathrm{~B}$ that at $T=134^{\circ} \mathrm{C}, \dot{\gamma}=2.5 \mathrm{~s}^{-1}$ corresponds closely to the critical condition for collapse of the shear stress, while the appearance and movement of occasional small bubbles at lower shear stresses is consistent

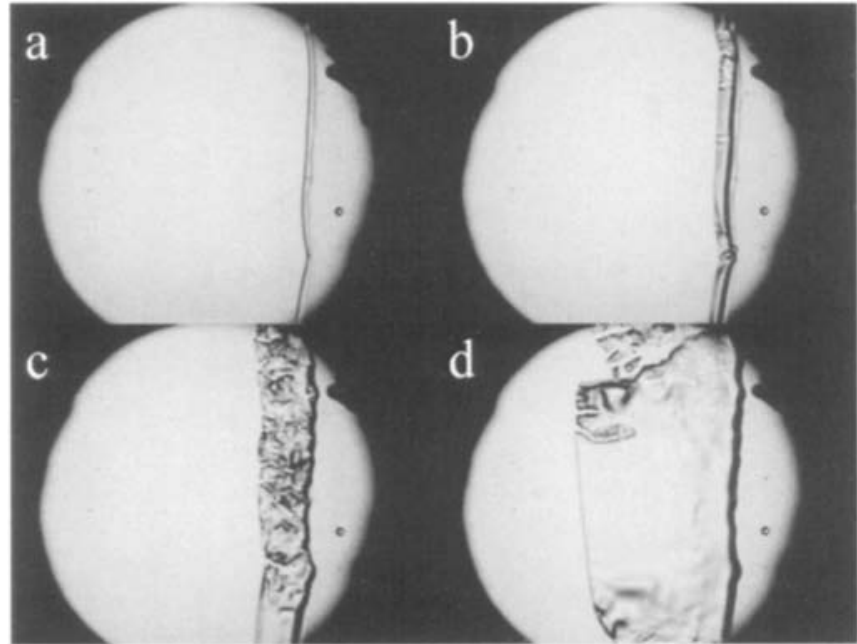

Fig. 7 As in Fig. 5, except that oscillatory shear is applied at a strain amplitude of 0.10 and a frequency of $1.6 \mathrm{~s}^{-1}$. The gap is $700 \mu \mathrm{m}$. The edge meniscus is at the right edge of the field of view. a) $0 \mathrm{~s}$; start of oscillatory shearing; b) $60 \mathrm{~s}$; the edge meniscus widens; c) $220 \mathrm{~s}$; the edge meniscus continues to widen and cracks; d) $860 \mathrm{~s}$; the meniscus is very wide

with the oscillatory stresses observed at these lower rates. The bubbles appeared to initiate near small dust particles in many cases. When the flow was turned off, the bubbles required several hours to slowly shrink and disappear.

Finally, to help explain the stress waveform decay observed in oscillatory shearing in the work of Chen et al., an oscillatory strain of $10 \%$ amplitude was applied to the polystyrene sample in the Cambridge shear cell at $134^{\circ} \mathrm{C}$. For frequencies ranging from 0.01 to $1.6 \mathrm{~s}^{-1}$, no bubbles were observed in the bulk of the sample. However, at the highest frequencies, $\omega \geq 1.0 \mathrm{~s}^{-1}$, the sample edge began to broaden, and at $1.6 \mathrm{~s}^{-1}$, pronounced cracking occurred within this broadened meniscus (see Fig. $7 \mathrm{a}-\mathrm{d}$ ). The formation of this broadened, cracked, meniscus correlates well with the observed damage near the edge of samples quenched after waveform decay had been observed in oscillatory shearing (Chen et al., 1994). The shear stress measured in such a sample is particularly sensitive to disruption at the sample edge, since the contribution to the torque signal grows with the third power of the radial position in the sample.

While it might be tempting to limit the relevance of these results to liquids near their glass transition temperature, the lack of temperature sensitivity of the critical stress contradicts that interpretation. For $\alpha$-D-glucose, the critical stress changed by at most a factor of two when the temperature was increased from $8^{\circ} \mathrm{C}$ to $22^{\circ} \mathrm{C}$ above $T_{g}$, while the critical shear rate increased by a factor of 30 (see Fig. 8). 


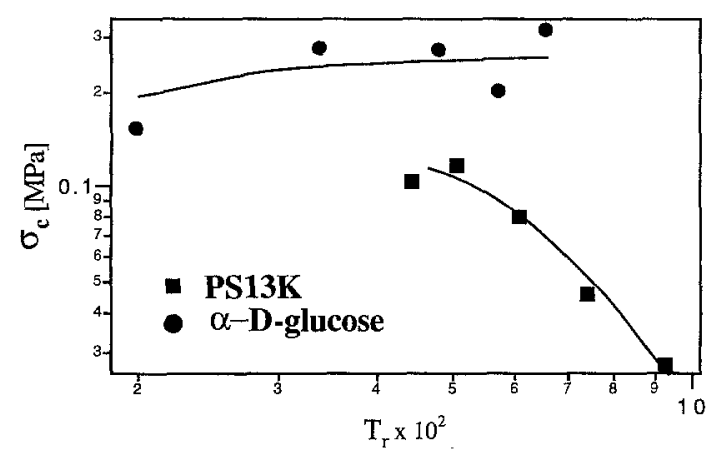

Fig. 8 The influence of reduced temperature, $T_{r} \equiv\left(T-T_{g}\right) / T_{g}$, on the magnitude of the critical "fracture" stress for $a$-D-glucose and PS13K. We take $T_{g}=308^{\circ} \mathrm{K}$ for $\alpha$-D-glucose and $370^{\circ} \mathrm{K}$ for polystyrene

\section{Discussion}

The results reported here have important implications for the flow of both small-molecule and polymeric fluids at high stresses. They prove that dramatic "fracture" phenomena can occur for fluids with Newtonian viscosities as low as $5 \times 10^{4} \mathrm{~Pa} \mathrm{~s}$, and suggest that they would also occur at much lower viscosities (obtained at higher temperatures) if the shear rates could be increased enough to generate the required shear stress of around 0.3 MPa. Indeed, Streator et al. (1986) recently showed that when perfluoropolyether lubricants with low viscosities in the range $0.05-2 \mathrm{~Pa}$ s were sheared at high rates in a thin-gap $(20-80 \mathrm{~nm})$ precision slider/disk geometry, there was a critical shear rate above which "lubricant rupture" occurred; i.e., the shear stress decreased with increasing shear rate. At low contact pressures, the critical shear stress $\sigma_{c}$ was around $0.2 \mathrm{MPa}$, and this value increased with increasing contact pressure $p$ roughly as $\sigma_{c}=\sigma_{0}+p$, where $\sigma_{0}$ is a constant, around $0.2 \mathrm{MPa}$. Similarly, a critical shear stress $\sigma_{c}$ somewhat in excess of the imposed hydrostatic pressure over the range $p=0.01-1.0 \mathrm{MPa}$ was observed in highstress shearing experiments in a circular Couette geometry by Winer and Bair (1990). Our experimental results are at least superficially similar to these, and the possible relationships between them are clearly worthy of further study.

\section{References}

Allen VR, Fox TG (1964) Viscosity-molecular weight dependence for short chain polystyrenes. J Chem Phys 41:337-343

Brochard F, de Gennes PG (1992) Shear-dependent slippage at a polymer/solid interface. Langmuir 8:3033-3037

Hatzikiriakos SG, Dealy JM (1991) Wall slip of molten high density polyethylene. I. Sliding plate rheometer studies. J Rheol 35:497-523

Chen Y-L, Larson RG, Patel SS (1994) Shear fracture of polystyrene melts and solutions. Rheol Acta 33:243-256
Larson RG (1988) Constitutive equations for polymer melts and solutions. Butterworths, Boston

Lee CS, Tripp BC, Magda JJ (1992) Does $N_{1}$ or $N_{2}$ control the onset of edge fracture? Rheol Acta 31:306-308

Migler KB, Hervet H, Leger L (1993) Slip transition of a polymer melt under shear stress. Phys Rev Lett 70:287-290

Streator JL, Gerhardstein JP, McCollum CB (1986) The low-pressure rheology of ultra-thin lubricant films and its influence on sliding contact. J Tribology 116:119126
Wang S-Q, Drda PA (1996) Superfluid-like stick-slip transition in capillary flow of linear polyethylene melts. 1. General features. Macromolecules 29:2627-2632

Winer WO, Bair SS (1990) The influence of ambient pressure on the apparent shear thinning of liquid lubricants - an overlooked phenomenon. J Tribology 112:246 